Bull. Korean Math. Soc. 50 (2013), No. 4, pp. 1209-1219

http://dx.doi.org/10.4134/BKMS.2013.50.4.1209

\title{
A NOTE ON THE HYPER-ORDER OF ENTIRE FUNCTIONS
}

\author{
FEng LÜ AND JiAnMing QI
}

\begin{abstract}
In the paper, we have two purposes. Firstly, we estimate the hyper-order of an entire function which shares two functions with it's first derivative, and two examples are given to show the conclusion is sharp. Secondly, we generalize the Brück conjecture with the idea of sharing functions.
\end{abstract}

\section{Introduction and main results}

In the Nevanlinna theory, the order and the hyper-order of a meromorphic function are two important concepts. So, it is meaningful to discuss the properties of the order and the hyper-order for a meromorphic function. Let us recall the definitions of the order and hyper-order of a meromorphic function $f$, which are respectively defined as (see [12])

$$
\begin{gathered}
\rho(f)=\limsup _{r \rightarrow \infty} \frac{\log T(r, f)}{\log r}=\limsup _{r \rightarrow \infty} \frac{\log \log M(r, f)}{\log r}, \\
\sigma(f)=\limsup _{r \rightarrow \infty} \frac{\log \log T(r, f)}{\log r}=\limsup _{r \rightarrow \infty} \frac{\log \log \log M(r, f)}{\log r} .
\end{gathered}
$$

We also define the hyper-lower-order as follows.

$$
\lambda(f)=\liminf _{r \rightarrow \infty} \frac{\log \log T(r, f)}{\log r}=\liminf _{r \rightarrow \infty} \frac{\log \log \log M(r, f)}{\log r} .
$$

In 1982, Bank and Laine [1] investigated the solutions of a differential equation and obtained the following result.

Theorem A. Let $A(z)$ be a nonconstant polynomial of degree $n$, and let $f \neq 0$ be a solution of the equation $f^{\prime \prime}+A(z) f=0$. Then the order of growth of $f$ is $\frac{n+2}{2}$.

Since then, to study properties of the order and hyper-order for the solutions of some differential equations becomes a hot topic and is discussed by many experts.

In 2008, Li and Gao [8] deduced a result as follows.

Received August 8, 2012.

2010 Mathematics Subject Classification. 30D35, 30D45.

Key words and phrases. complex differential equation, normal family, Nevanlinna theory. 
Theorem B. Let $Q_{1}$ and $Q_{2}$ be two nonzero polynomials, and let $P$ be a polynomial. If $f$ is a nonconstant solution of the equation

$$
f^{(k)}-Q_{1}=e^{P}\left(f-Q_{2}\right),
$$

then $\sigma(f)=n$, where, and in the sequel, $n$ denotes the degree of $P$.

It is well-known that the problem of the differential equations is closely related with the problem of meromorphic functions sharing values. So, it is an interesting attempt to derive the properties of order and hyper-order for meromorphic functions by considering the situation of sharing values.

In 2009, Lü and Yi [11] obtained a result.

Theorem C. Let $f$ be a nonconstant meromorphic function with finitely many poles, and let $Q_{1}, Q_{2}\left(\neq Q_{1}\right)$ be two polynomials. If

$$
f(z)=Q_{1} \Rightarrow f^{\prime}(z)=Q_{1} \text { and } f(z)=Q_{2} \Rightarrow f^{\prime}(z)=Q_{2},
$$

then $f$ is of finite order.

In 2009, Chen, Lü and Yi [4] improved the above theorem and deduced the following result.

Theorem D. Let $f$ be a nonconstant meromorphic function with finitely many poles, and let $R_{3}(z)=c_{1} e^{z}$ and $R_{4}(z)=c_{2} e^{z}$, where $c_{1}, c_{2}$ are two nonzero constants. If

$$
f(z)=R_{3} \Rightarrow f^{\prime}(z)=R_{3} \text { and } f(z)=R_{4} \Rightarrow f^{\prime}(z)=R_{4},
$$

then $f$ is of finite order.

From Theorem $\mathrm{C}$ and $\mathrm{D}$, we see that $f$ and $f^{\prime}$ share two functions with finite order. So, it is natural to ask what will happen if they share functions with infinite order. In the work, we discuss the problem and derive the following result.

Theorem 1.1. Let $Q_{1} \neq 0$ and $Q_{2}$ be two distinct polynomials, let $f, \gamma$ be two entire functions. If

$$
f(z)=\alpha(z) \Rightarrow f^{\prime}(z)=\alpha(z) \text { and } f(z)=\beta(z) \Rightarrow f^{\prime}(z)=\beta(z),
$$

where $\alpha=Q_{1} e^{\gamma}, \beta=Q_{2} e^{\gamma}$, and if $\alpha-\alpha^{\prime}$ or $\beta-\alpha^{\prime}$ has at most finitely many zeros, then $\sigma(f) \leq \sigma(\alpha)=\rho(\gamma)$.

Remark 1. The following examples show that the conclusion $\sigma(f) \leq \rho(\gamma)$ is sharp.

Example 1. Let $f(z)=A e^{z}$, where $A$ is a nonzero constant. Let $\alpha(z)=$ $e^{e^{-z}+z}$, and let $\beta(z)=P(z) e^{e^{-z}+z}$, where $P(z)$ is a polynomial. Noting that $f=f^{\prime}$, we have

$$
f(z)=\alpha(z) \Rightarrow f^{\prime}(z)=\alpha(z) \text { and } f(z)=\beta(z) \Rightarrow f^{\prime}(z)=\beta(z) .
$$

Obviously, $\alpha(z)-\alpha^{\prime}(z)=e^{e^{-z}}$ has no zeros. Thus, it satisfies the assumptions of Theorem 1.1 and $\sigma(f)=0<\sigma(\alpha)=1$. 
Example 2. Let $f(z)=z e^{z^{2}}+e^{z / 2}, \alpha(z)=\left(4 z^{2}-z+2\right) e^{z^{2}}$, and $\beta(z)=z e^{z^{2}}$. Then

$$
\frac{f^{\prime}-\alpha}{f-\alpha}=\frac{1}{2} \text { and } f(z)-\beta(z) \neq 0 .
$$

It satisfies the assumptions of Theorem 1.1 and $\sigma(f)=0$.

Remark 2. The condition that $\alpha-\alpha^{\prime}$ or $\beta-\alpha^{\prime}$ has at most finitely many zeros is essential in our proof of Theorem 1.1. But we don't know whether it is necessary or not. If $\gamma$ is a polynomial, then the above condition obviously holds.

Remark 3. In Theorem 1.1, if the order of $\gamma$ is zero, for example $\gamma$ is a polynomial, then $\sigma(f)=0$, which is an important property for a meromorphic function $f$.

Remark 4. If $f$ is a meromorphic function with finitely many poles and $Q_{1}, Q_{2}$ are two rational functions, the conclusion is still valid in Theorem 1.1.

\section{Some results of the Brück conjecture}

In 1996, Brück [3] posed the following conjecture which reveals the relationship between $f$ and $f^{\prime}$ if an entire function $f$ shares one value CM with its first derivative $f^{\prime}$ ?

Conjecture. Let $f$ be a non-constant entire function such that the hyperorder $\sigma(f)$ of $f$ is not a positive integer and $\sigma(f)<\infty$. If $f$ and $f^{\prime}$ share a finite value $a \mathrm{CM}$, then

$$
\frac{f^{\prime}-a}{f-a}=c
$$

where $c$ is non-zero constant.

In [5], Chen generalized the Brück conjecture with the idea of sharing $z$ and got the following result.

Theorem E. Let $f$ be a nonconstant entire function such that the hyper order $\sigma(f)<\frac{1}{2}, k$ be a positive integer. If $f$ and $f^{(k)}$ share $z C M$, then

$$
f^{(k)}(z)-z=c(f(z)-z),
$$

where $c$ is a nonzero constant.

In the following, due to the methods of Chen and Zhang [5], Zhang and Liao [13], we obtain a result which is an improvement of the previous theorems in some sense.

Theorem 2.1. Let $f, \alpha$ be two meromorphic functions with at most finitely many poles, and let $\sigma(f)<\frac{1}{2}$ and $\sigma(\alpha)<\lambda(f)$. If $f$ and $\alpha$ have no common poles, $f$ and $f^{(k)}$ share $\alpha C M$, then $\frac{f^{(k)}-\alpha}{f-\alpha}=c$, where $c$ is a nonzero constant. 
Remark 5. In the proof of Theorem 2.1, we need the condition $\sigma(P)<\lambda(f)$ to confirm that $r \notin E$

$$
\frac{M(r, P)}{M(r, f)} \rightarrow 0 \text { as } r \rightarrow \infty,
$$

where $E \subset[0,+\infty)$ is a set with finite logarithmic measure. If it is deleted, we do not know whether the above conclusion holds or not.

Remark 6. If $P$ is a polynomial, then the condition $\sigma(P)<\lambda(f)$ can be deleted in Theorem 2.1. The result is listed as below, which can be proved with the same way in Theorem 2.1.

Corollary 2.2. Let $f$ be a meromorphic function with at most finitely many poles and hyper-order $\sigma(f)<\frac{1}{2}$, let $P$ be a non-zero polynomial. If $f$ and $f^{(k)}$ share $P C M$, then $\frac{f^{(k)}-P}{f-P}=c$, where $c$ is a nonzero constant.

\section{Some lemmas}

In order to prove our theorems, we need the following lemmas.

Lemma 3.1 ([10, 9]). Let $\left\{f_{n}\right\}$ be a family of functions meromorphic (analytic) on the unit disc $\triangle$. If $a_{n} \rightarrow a,|a|<1$, and $f_{n}^{\sharp}\left(a_{n}\right) \rightarrow \infty$, then there exist

(a) a subsequence of $f_{n}$ (which we still write as $\left.\left\{f_{n}\right\}\right)$;

(b) points $z_{n} \rightarrow z_{0}\left|z_{0}\right|<1$;

(c) positive numbers $\rho_{n} \rightarrow 0$

such that $f_{n}\left(z_{n}+\rho_{n} \xi\right)=g_{n}(\xi) \rightarrow g(\xi)$ locally uniformly, where $g$ is a nonconstant meromorphic (entire) function on $\mathbb{C}$, such that

$$
\rho_{n} \leq \frac{M}{f_{n}^{\sharp}\left(a_{n}\right)},
$$

where $M$ is a constant which is independent of $n$.

With a similar method in [9, Lemma 2], we obtain the following result, which plays an important part in the proof of Theorem 1.1.

Lemma 3.2. Let $f$ be a meromorphic function of hyper-order $\sigma(f)>0$. Then, for any $\epsilon>0$, there exists a sequence $z_{n} \rightarrow \infty$ such that $f^{\sharp}\left(z_{n}\right)>e^{\left|z_{n}\right|^{\sigma(f)-\epsilon}}$ if $n$ is large enough.

Proof. On the contrary, there exist $\epsilon>0, N>0$ and $R>0$ such that for all $z,|z| \geq R$ satisfying $f^{\sharp}(z) \leq e^{|z|^{\sigma(f)-\epsilon}}$. Thus,

$$
\begin{aligned}
S(r, f) & =\frac{1}{\pi} \iint_{|z|<r} f^{\sharp}(z)^{2} d \sigma=\frac{1}{\pi} \iint_{R \leq|z|<r} f^{\sharp}(z)^{2} d \sigma+O(1) \\
& \leq \frac{1}{\pi} \iint_{R \leq|z|<r} e^{2|z|^{\sigma(f)-\epsilon}} d \sigma+O(1)=\frac{1}{\pi} \int_{0}^{2 \pi} d \theta \int_{R}^{r} e^{2|z|^{\sigma(f)-\epsilon}} d t+O(1) \\
& \leq 2 r e^{2 r^{\sigma(f)-\epsilon}}[1+o(1)] .
\end{aligned}
$$


By the definition of Ahlfors characteristic of $f$, we have

$$
T(r, f)=\int_{0}^{r} \frac{S(t, f)}{t} d t \leq 2 r e^{2 r^{\sigma(f)-\epsilon}}[1+o(1)] .
$$

Then, the hyper-order of $f$ is

$$
\sigma(f)=\limsup _{r \rightarrow \infty} \frac{\log \log T(r, f)}{\log r} \leq \limsup _{r \rightarrow \infty} \frac{(\sigma(f)-\epsilon) \log r}{\log r}=\sigma(f)-\epsilon,
$$

a contradiction. Thus, the proof is completed.

Lemma $3.3([2])$. Let $h(z)$ be an entire function with order $\rho(h)=\rho<\frac{1}{2}$, set

$$
A(r)=\inf _{|z|=r} \log |h(z)|, B(r)=\sup _{|z|=r} \log |h(z)| .
$$

If $\rho<\alpha<1$, then

$$
\underline{\log \operatorname{dens}}\{r: A(r)>(\cos \pi \alpha) B(r)\} \geq 1-\frac{\rho}{\alpha}
$$

where the lower logarithmic density $\underline{\log \text { dens } H}$ of subset $H \subset(1, \infty)$ is defined by

$$
\underline{\log \operatorname{dens}} H=\liminf \left(\int_{1}^{r}\left(\mathcal{X}_{H}(t) / t\right) d t\right) / \log r,
$$

where $\mathcal{X}_{H}(t)$ is the characteristic function of set $H$.

Remark 7. From the definitions of logarithmic measure and logarithmic density, it is easy to see that the lower logarithmic density $\log$ dens $H>0$, then the logarithmic measure $l m H=+\infty$.

Lemma 3.4 ([7, p. 51 Theorem 3.2]). Let $f$ be a transcendental entire function and $0<\delta<\frac{1}{4}$. Suppose that at the point $z$ with $|z|=r$ the inequality

$$
|f(z)|>M(r, f) \nu(r, f)^{-\frac{1}{4}+\delta}
$$

holds, then there exists a set $F \in R^{+}$of finite logarithmic measure, i.e., $\int_{F} \frac{1}{t} d t<+\infty$, such that

$$
f^{(m)}(z)=\left(\frac{\nu(r, f)}{z}\right)^{m}(1+o(1)) f(z)
$$

holds for all $r \notin F$.

\section{The Proof of Theorem 1.1}

With the similar way of $[6,10]$, we prove Theorem 1.1 as follows.

Noting that $\alpha=Q_{1} e^{\gamma}$, thus $\sigma(\alpha)=\rho(\gamma)$. So, we just need to obtain $\sigma(f) \leq \rho(\gamma)$

On the contrary, assume that $\sigma(f)=d>c=\rho(\gamma)$. Set $H=f-\alpha$. Then

(I) $H(z)=0 \Rightarrow H^{\prime}(z)=\alpha(z)-\alpha^{\prime}(z)$,

(II) $H(z)=\beta(z)-\alpha(z) \Rightarrow H^{\prime}(z)=\beta(z)-\alpha^{\prime}(z)$. 
Let $P=\beta-\alpha$ and $F=\frac{H}{P}$. Obviously, $\sigma(F)=\sigma(f)=d$. By Lemma 3.2, then for $0<\epsilon<\frac{d-c}{2}$, there exists a sequence $w_{n} \rightarrow \infty$ as $n \rightarrow \infty$ such that

$$
F^{\sharp}\left(w_{n}\right)>e^{\left|w_{n}\right|^{\sigma(F)-\epsilon}}=e^{\left|w_{n}\right|^{d-\epsilon}} .
$$

Noting that $P=\beta-\alpha$ has at most finitely many zeros, then there exists a positive number $r$ such that $F$ has no poles in $D=\{z:|z|>r\}$.

In view of $w_{n} \rightarrow \infty$ as $n \rightarrow \infty$, without loss of generality, we may assume $\left|w_{n}\right| \geq r+1$ for all $n$. Define $D_{1}=\{z:|z|<1\}$ and

$$
F_{n}(z)=F\left(w_{n}+z\right) \text {. }
$$

Then every $F_{n}$ are analytic in $D_{1}$ and $F_{n}^{\sharp}(0) \rightarrow \infty$ as $n \rightarrow \infty$. It follows from Marty's criterion that $\left(F_{n}\right)_{n}$ is not normal at $z=0$.

Therefore, we can apply Lemma 3.1. Choosing an appropriate subsequence of $\left(F_{n}\right)_{n}$ if necessary, we may assume that there exist sequences $\left(z_{n}\right)_{n}$ and $\left(\rho_{n}\right)_{n}$ with $\left|z_{n}\right|<r<1$ and $\rho_{n} \rightarrow 0$ such that the sequence $\left(g_{n}\right)_{n}$ defined by

$$
g_{n}(\zeta)=F_{n}\left(z_{n}+\rho_{n} \zeta\right)=\frac{H\left(w_{n}+z_{n}+\rho_{n} \zeta\right)}{P\left(w_{n}+z_{n}+\rho_{n} \zeta\right)} \rightarrow g(\zeta)
$$

locally uniformly in $\mathbb{C}$ with a nonconstant entire function $g$ and

$$
\rho_{n} \leq \frac{M}{F_{n}^{\sharp}(0)}=\frac{M}{F^{\sharp}\left(w_{n}\right)} \leq M e^{-\left|w_{n}\right|^{d-\epsilon}}
$$

for a positive number $M$.

From (4.1), we have

$$
\begin{aligned}
g_{n}^{\prime}(\zeta) & =\rho_{n} \frac{H^{\prime}\left(w_{n}+z_{n}+\rho_{n} \zeta\right)}{P\left(w_{n}+z_{n}+\rho_{n} \zeta\right)}-\rho_{n} \frac{H\left(w_{n}+z_{n}+\rho_{n} \zeta\right) P^{\prime}\left(w_{n}+z_{n}+\rho_{n} \zeta\right)}{P^{2}\left(w_{n}+z_{n}+\rho_{n} \zeta\right)} \\
& \rightarrow g^{\prime}(\zeta) .
\end{aligned}
$$

Noting that $P=\alpha-\beta=Q e^{\gamma}$, where $Q=Q_{1}-Q_{2}$ is a nonzero polynomial, we have $\frac{P^{\prime}}{P}=\frac{Q^{\prime}+Q \gamma^{\prime}}{Q}$ and $\rho\left(\gamma^{\prime}\right)=\rho(\gamma)=c$. In view of the definition of order, we have

$$
\begin{aligned}
\left|\frac{P^{\prime}}{P}\right|_{z=w_{n}+z_{n}+\rho_{n} \zeta} \mid & =\left|\frac{Q^{\prime}+Q \gamma^{\prime}}{Q}\right|_{z=w_{n}+z_{n}+\rho_{n} \zeta} \mid \\
& \leq\left|w_{n}\right|^{q} M\left(\left|w_{n}+z_{n}+\rho_{n} \zeta\right|, \gamma^{\prime}\right) \\
& \leq\left|w_{n}\right|^{q} M\left(2\left|w_{n}\right|, \gamma^{\prime}\right) \leq\left|w_{n}\right|^{q} e^{A\left|w_{n}\right|^{c+\epsilon}}
\end{aligned}
$$

where $A$ is a positive constant and $q$ is an integer. Noting that $0<\epsilon<\frac{d-c}{2}$, we have $d-\epsilon>c+\epsilon$. Then, combining (4.2) and (4.4) yields

$$
\begin{aligned}
& \quad\left|\rho_{n} \frac{H\left(w_{n}+z_{n}+\rho_{n} \zeta\right) P^{\prime}\left(w_{n}+z_{n}+\rho_{n} \zeta\right)}{P^{2}\left(w_{n}+z_{n}+\rho_{n} \zeta\right)}\right|=\left|\rho_{n} g_{n}(\zeta) \frac{P^{\prime}\left(w_{n}+z_{n}+\rho_{n} \zeta\right)}{P\left(w_{n}+z_{n}+\rho_{n} \zeta\right)}\right| \\
& \leq M\left|g_{n}(\zeta)\right|\left|w_{n}\right|^{q} e^{A\left|w_{n}\right|^{c+\epsilon}-\left|w_{n}\right|^{d-\epsilon}} \rightarrow 0 \text { as } n \rightarrow \infty .
\end{aligned}
$$


From (4.3) and (4.5), we deduce that

$$
\rho_{n} \frac{H^{\prime}\left(w_{n}+z_{n}+\rho_{n} \zeta\right)}{P\left(w_{n}+z_{n}+\rho_{n} \zeta\right)} \rightarrow g^{\prime}(\zeta)
$$

We claim that

(1) $g(\zeta)=0 \Rightarrow g^{\prime}(\zeta)=0$ and

(2) $g(\zeta)=1 \Rightarrow g^{\prime}(\zeta)=0$.

Suppose that $g\left(\zeta_{0}\right)=0$, then by Hurwitz's theorem there exist $\zeta_{n}, \zeta_{n} \rightarrow \zeta_{0}$, such that (for $n$ sufficiently large)

$$
g_{n}\left(\zeta_{n}\right)=\frac{H\left(w_{n}+z_{n}+\rho_{n} \zeta_{n}\right)}{P\left(w_{n}+z_{n}+\rho_{n} \zeta_{n}\right)}=0 .
$$

By the assumption of (I) we have

$$
H^{\prime}\left(w_{n}+z_{n}+\rho_{n} \zeta_{n}\right)=\left(\alpha-\alpha^{\prime}\right)\left(w_{n}+z_{n}+\rho_{n} \zeta_{n}\right) .
$$

Observing that $\alpha-\alpha^{\prime}=\left(Q_{1}-Q_{1}^{\prime}-Q_{1} \gamma^{\prime}\right) e^{\gamma}$, similarly as (4.5), we have

$$
\begin{aligned}
& \rho_{n}\left|\frac{\left(\alpha-\alpha^{\prime}\right)\left(w_{n}+z_{n}+\rho_{n} \zeta_{n}\right)}{P\left(w_{n}+z_{n}+\rho_{n} \zeta_{n}\right)}\right| \\
= & \rho_{n}\left|\frac{\left[Q_{1}-Q_{1}^{\prime}-Q_{1} \gamma^{\prime}\right]\left(w_{n}+z_{n}+\rho_{n} \zeta_{n}\right)}{\left[Q_{1}-Q_{2}\right]\left(w_{n}+z_{n}+\rho_{n} \zeta_{n}\right)}\right| \rightarrow 0 \text { as } n \rightarrow \infty .
\end{aligned}
$$

Then,

$g^{\prime}\left(\zeta_{0}\right)=\lim _{n \rightarrow \infty} \rho_{n} \frac{H^{\prime}\left(w_{n}+z_{n}+\rho_{n} \zeta_{n}\right)}{P\left(w_{n}+z_{n}+\rho_{n} \zeta_{n}\right)}=\lim _{n \rightarrow \infty} \rho_{n} \frac{\left(\alpha-\alpha^{\prime}\right)\left(w_{n}+z_{n}+\rho_{n} \zeta_{n}\right)}{P\left(w_{n}+z_{n}+\rho_{n} \zeta_{n}\right)}=0$.

Thus $g(\zeta)=0 \Rightarrow g^{\prime}(\zeta)=0$, which is (1). Similarly, we can prove (2). Thus, we finish the proof of the claims.

Noting that either $\alpha-\alpha^{\prime}$ or $\beta-\alpha^{\prime}$ has at most finitely many zeros, without loss of generality, we assume that $\alpha-\alpha^{\prime}$ has at most finitely many zeros. Next, we shall prove that $g(\zeta) \neq 0$.

Suppose $\xi_{0}$ is a zero of $g(\zeta)$ with multiplicity $m(\geq 2)$. Then $g^{(m)}\left(\xi_{0}\right) \neq 0$. Thus there exists a positive number $\delta$, such that

$$
g(\zeta) \neq 0, g^{\prime}(\zeta) \neq 0, g^{(m)}(\zeta) \neq 0
$$

on $D_{\delta}^{o}=\left\{z: 0<\left|\zeta-\xi_{0}\right|<\delta\right\}$.

Noting that $g \neq 0$, by Rouché theorem there exist $\zeta_{n, j}(j=1,2, \ldots, m)$ on $D_{\delta / 2}=\left\{\xi:\left|\zeta-\xi_{0}\right|<\delta / 2\right\}$ such that

$$
g_{n}\left(\zeta_{n, j}\right)=H\left(w_{n}+z_{n}+\rho_{n} \zeta_{n, j}\right)=0(j=1, \ldots, m) .
$$

Observing that $\alpha-\alpha^{\prime}$ has at most finitely many zeros, then (for $n$ large enough) we have

$$
\begin{aligned}
& H^{\prime}\left(w_{n}+z_{n}+\rho_{n} \zeta_{n, j}\right) \\
= & {\left[\alpha-\alpha^{\prime}\right]\left(w_{n}+z_{n}+\rho_{n} \zeta_{n, j}\right) \neq 0, }
\end{aligned}
$$

which implies that each $\zeta_{n, j}$ is a simple zero of $H\left(w_{n}+z_{n}+\rho_{n} \zeta\right)$, that is $\zeta_{n, j} \neq \zeta_{n, i}(1 \leq i \neq j \leq m)$. 
As in (4.5), we deduce

$$
\rho_{n} \frac{\left[\alpha-\alpha^{\prime}\right]\left(w_{n}+z_{n}+\rho_{n} \zeta\right)}{P\left(w_{n}+z_{n}+\rho_{n} \zeta\right)} \rightarrow 0 .
$$

It follows from (4.6) and (4.9) that

$$
K_{n}(\zeta)=\rho_{n} \frac{H^{\prime}\left(w_{n}+z_{n}+\rho_{n} \zeta\right)}{P\left(w_{n}+z_{n}+\rho_{n} \zeta\right)}-\rho_{n} \frac{\left[\alpha-\alpha^{\prime}\right]\left(w_{n}+z_{n}+\rho_{n} \zeta\right)}{P\left(w_{n}+z_{n}+\rho_{n} \zeta\right)} \rightarrow g^{\prime}(\zeta)
$$

and $K_{n}\left(\zeta_{n, j}\right)=0(j=1, \ldots, m)$. By $(4.8)$ we obtain

$$
\lim _{n \rightarrow \infty} \zeta_{n, j}=\xi_{0}(j=1,2, \ldots, m) \text {. }
$$

Noting that (4.8), (4.10) and $K_{n}(\zeta)$ has $m$ zeros $\zeta_{n, j}(j=1,2 \ldots, m)$ in $D_{\delta / 2}$, we obtain from the Hurwitz's theorem that $\xi_{0}$ is a zero of $g^{\prime}$ with multiplicity $m$, and thus $g^{(m)}\left(\xi_{0}\right)=0$. This is a contradiction. Hence $g(\zeta) \neq 0$.

We have shown that $g$ is a non-vanishing, entire function that takes the value 1 always with multiplicity at least 2 . But this contradicts Nevanlinnna's second fundamental theorem that the sum of the defects is at most 2 .

Thus, we complete the proof of Theorem 1.1.

\section{Proof of Theorem 2.1}

From the assumptions of Theorem 2.1, we set

$$
\frac{f^{(k)}-\alpha}{f-\alpha}=\frac{1}{Q} e^{\phi}
$$

where $\phi$ is an entire function and $Q$ is a polynomial. Noting that $f$ and $\alpha$ have only finitely many poles, then there exists a polynomial $P$ such that $f P$ and $\alpha P$ are two entire functions. Set

$$
F=f P \text { and } A=\alpha P \text {. }
$$

Obviously, $\sigma(F)=\sigma(f)<\frac{1}{2}$ and $\sigma(A)=\sigma(\alpha)<\lambda(F)=\lambda(f)$. We also have

$$
f^{(k)}=\left(\frac{F}{P}\right)^{(k)}=\sum_{j=0}^{k} C_{k, j} F^{(k-j)}\left(\frac{1}{P}\right)^{(j)},
$$

where $C_{k, j}=\frac{k !}{j !(k-j) !}$ is a constant.

In the following, we will prove $\phi$ is a constant.

Rewriting (5.1) as

$$
\begin{aligned}
e^{\phi} & =Q \frac{f^{(k)}-\alpha}{f-\alpha}=Q \frac{\sum_{j=0}^{k} C_{k, j} F^{(k-j)}\left(\frac{1}{P}\right)^{(j)}-\frac{A}{P}}{\frac{F}{P}-\frac{A}{P}} \\
& =Q \frac{\sum_{j=0}^{k} C_{k, j} \frac{F^{(k-j)}}{F}\left(\frac{1}{P}\right)^{(j)} P-\frac{A}{F}}{1-\frac{A}{F}} .
\end{aligned}
$$


From (5.3), we have

$$
\phi=\log Q \frac{\sum_{j=0}^{k} C_{k, j} \frac{F^{(k-j)}}{F}\left(\frac{1}{P}\right)^{(j)} \frac{1}{P}-\frac{A}{F}}{1-\frac{A}{F}},
$$

where $\log g$ is a principle branch of $\log g$. By Lemma 3.4, we get

$$
\frac{F^{(k-j)}\left(z_{r}\right)}{F\left(z_{r}\right)}=\left(\frac{\nu(r, F)}{z_{r}}\right)^{k-j}(1+o(1))(j=0, \ldots, k)
$$

possibly outside a set of finite logarithmic measure $E$, where $\left|F\left(z_{r}\right)\right|=M(r, F)$. Observing that $\sigma(A)<\lambda(F)$, for $0<\epsilon<\frac{\lambda(F)-\sigma(A)}{2}$, we have

$$
\sigma(A)=\limsup _{r \rightarrow \infty} \frac{\log \log \log M(r, A)}{\log r} \leq \sigma(A)+\epsilon,
$$

which implies that (for $r$ large enough)

$$
M(r, A) \leq e^{e^{r^{\sigma(A)+\epsilon}}} .
$$

Similarly, we deduce that (for $r$ large enough)

$$
M(r, F) \geq e^{e^{r^{\lambda(F)-\epsilon}}} .
$$

Observing that $\epsilon<\frac{\lambda(F)-\sigma(A)}{2}$, we have $\sigma(A)+\epsilon<\lambda(F)-\epsilon$. Then, combining (5.6) and (5.7) yields that

$$
\begin{aligned}
\frac{M(r, A)}{M(r, F)} & \leq \frac{e^{e^{r^{\sigma(A)+\epsilon}}}}{e^{e^{r^{\lambda(F)-\epsilon}}}} \\
& \leq \frac{1}{e^{\left[e^{r^{\lambda(F)-\epsilon}}-e^{r^{\sigma(A)+\epsilon}}\right]} \rightarrow 0 \text { as } r \rightarrow \infty} .
\end{aligned}
$$

It indicates that

$$
\frac{M(r, A)}{M(r, F)} \rightarrow 0 \text { as } r \rightarrow \infty .
$$

So, substituting (5.5) (5.9) into (5.4), we deduce

$$
\begin{aligned}
\left|\phi\left(z_{r}\right)\right| & =\left|\log Q \frac{\sum_{j=0}^{k} C_{k, j} \frac{F^{(k-j)}}{F}\left(\frac{1}{P}\right)^{(j)} P-\frac{A}{F}}{1-\frac{A}{F}}\right| \\
& \leq|\log | Q \frac{\sum_{j=0}^{k} C_{k, j} \frac{F^{(k-j)}}{F}\left(\frac{1}{P}\right)^{(j)} P-\frac{A}{F}}{1-\frac{A}{F}}||+2 \pi \\
& \leq A[1+o(1)] \log \nu(r, F),
\end{aligned}
$$

for $\left|z_{r}\right|=r \notin E$, where $A$ is a positive number. Now, we consider into two cases.

Case 1. $\phi$ is a polynomial with degree $n \geq 1$.

Noting that the definition of hyper-order of $f$, for $\epsilon<\frac{1}{2}$, we have

$$
\log \nu(r, F) \leq r^{\left(\frac{1}{2}+\epsilon\right)} .
$$


Then, from (5.10) we obtain that

$$
B(1+o(1))|r|^{n}=\left|\phi\left(z_{r}\right)\right| \leq A[1+o(1)] r^{\left(\frac{1}{2}+\epsilon\right)},
$$

where $B$ is a positive constant. This is a contradiction.

Case 2. $\phi$ is a transcendental entire function.

Since $\frac{\log \log \nu(r, F)}{\log r}<\frac{1}{2}$ for sufficiently large $r$, from (5.10),

$$
\left|\phi\left(z_{r}\right)\right| \leq A[1+o(1)] r^{\frac{1}{2}} .
$$

Obviously, $\rho(\phi) \leq \sigma(f)<\frac{1}{2}$. Then, by Lemma 3.3, we know that there exists a set $H \subset(1, \infty)$ that have logarithmic measure $\operatorname{lm} H=\infty$, such that for all $|z|=r \in H$, we have

$$
|\phi(z)| \geq M(r, \phi)^{c},
$$

where $0<c<1$. Now, for all $z_{r}$ satisfying $\left|z_{r}\right|=r \in H \backslash(E \bigcup[0,1])$ and $\left|F\left(z_{r}\right)\right|=M(r, F)$, by (5.13) and (5.14), we get

$$
\frac{M(r, \phi)^{c}}{r^{\frac{1}{2}}} \leq A
$$

Since $\phi$ is transcendental, we derive that

$$
\frac{M(r, \phi)^{c}}{r^{\frac{1}{2}}} \rightarrow \infty
$$

which contradicts with (5.15). Thus, $\phi$ is a constant.

Now, in the same way as [13, Theorem 1.1] we prove that $Q$ is a constant. On the contrary, we suppose that $q=\operatorname{deg} Q \geq 1$ and $e^{\phi}=1$ without loss of generality.

Rewriting (5.9) as

$$
1=Q \frac{\sum_{j=0}^{k} C_{k, j} \frac{F^{(k-j)}}{F}\left(\frac{1}{P}\right)^{(j)} P-\frac{A}{F}}{1-\frac{A}{F}} .
$$

Since $P$ is a polynomial, we have

$$
\left|\left(\frac{1}{P}\right)^{(j)}\left(z_{r}\right) P\left(z_{r}\right)\right|=r^{-j}\left(d_{1}+o(1)\right)
$$

where $d_{1}$ is a positive number. We know that any zero of $Q$ comes from a pole of $f$ with multiplicity $k$. Thus $q \geq k$. Then, from the equations (5.5), (5.9), and (5.17) we derive that $(|z|=r \notin E)$

$$
r^{q-k} \nu(r, F)^{k}\left(d_{2}+o(1)\right)=1,
$$

where $d_{2}$ is a positive number. Thus, it implies that $\nu(r, F)$ is bounded. While, for a transcendental entire function $F$, we have

$$
\nu(r, F) \rightarrow \infty \text { as } r \rightarrow \infty .
$$

So, we derive a contradiction. Hence, $Q$ is also a constant, and $\frac{e^{\phi}}{Q}$ becomes a constant $c$.

Hence, we complete the proof of Theorem 2.1. 
Acknowledgements. We are grateful to the reviewers for their helpful comments and suggestions. The research was supported by the Natural Science Foundation of Shandong Province Youth Fund Project (ZR2012AQ021), the Fundamental Research Funds for the Central Universities (12CX04080A), the Shanghai university young teacher training program(ZZSDJ12020), project 10XKJ01, 12C401 and 12C104 from the Leading Academic Discipline Project of Shanghai Dianji University.

\section{References}

[1] S. Bank and I. Laine, On the oscillation theory of $f^{\prime \prime}+A(z) f=0$ where $A$ is entire, Trans. Amer. Math. Soc. 273 (1982), no. 1, 351-363.

[2] P. D. Barry, On a theorem of Besicovitch, Quart. J. Math. Oxford Ser. (2) 14 (1963), 293-320.

[3] R. Brück, On entire functions which share one value CM with their first derivatives, Results Math. 30 (1996), no. 1-2, 21-24.

[4] A. Chen, F. Lü, and H. X. Yi, Value sharing of meromorphic functions and their derivatives, J. Math. Anal. Appl. 359 (2009), no. 2, 696-703.

[5] Z. X. Chen and Z. L. Zhang, Entire functions sharing fixed points with their higher order derivatives, Acta Math. Sinica (Chin. Ser.) 50 (2007), no. 6, 1213-1222.

[6] J. Grahl and C. Meng, Entire functions sharing a polynomial with their derivatives and normal families, Analysis (Munich) 28 (2008), no. 1, 51-61.

[7] I. Laine, Nevanlinna Theory and Complex Differential Equations, de Gruyter Studies in Mathematics, 15. Walter de Gruyter \& Co., Berlin, 1993.

[8] X. M. Li and C. C. Gao, Entire functions sharing one polynomial with their derivatives, Proc. Indian Acad. Sci. Math. Sci. 118 (2008), no. 1, 13-26.

[9] X. J. Liu, S. Nevo, and X. C. Pang, On the kth derivative of meromorphic functions with zeros of multiplicity at least $k+1$, J. Math. Anal. Appl. 348 (2008), no. 1, 516-529.

[10] F. Lü, J. F. Xu, and A. Chen, Entire functions sharing polynomials with their first derivatives, Arch. Math. (Basel) 92 (2009), no. 6, 593-601.

[11] F. Lü and H. X. Yi, On the uniqueness problems of meromorphic functions and their linear differential polynomials, J. Math. Anal. Appl. 362 (2010), no. 2, 301-312.

[12] H. X. Yi and C. C. Yang, Uniqueness Theory of Meromorphic Functions, Science Press, Beijing, 1995.

[13] J. Zhang and L. W. Liao, On Brück's conjecture on entire functions sharing one value with their derivatives, Houston. J. Math. 36 (2010), no. 2, 665-674.

FENG Lü

College of Science

China University of Petroleum

QingdaO, 266580, P. R. China

E-mail address: lvfeng18@gmail.com

JIANMING QI

Department of Mathematics And Physics

SHANGHAI DIANJI UNIVERSITY

Shanghai, 201306, P. R. ChinA

E-mail address: qijianmingdaxia@163.com 